\title{
Evaluating Usefulness of Maine's Syndromic Surveillance System for Hospitals, 2012
}

\author{
Stefanie DeVita* and Amy Robbins \\ Infectious Disease Epidemiology, Maine CDC, Augusta, ME, USA
}

\section{Objective}

To assess the usefulness and acceptability of Maine's syndromic surveillance system among hospitals who currently participate.

\section{Introduction}

Maine has been conducting syndromic surveillance since 2007 using the Early Aberration Reporting System (EARS). An evaluation of the syndromic surveillance system was conducted to determine if system objectives are being met, assess the system's usefulness, and identify areas for improvement. According to CDC's Guidelines for Evaluating Public Health Surveillance Systems, a surveillance system is useful if it contributes to the timely prevention and control of adverse health events. Acceptability includes the willingness of participants to report surveillance data; participation or reporting rate; and completeness of data.

\section{Methods}

A survey was created in 2012 to measure usefulness and acceptability among hospital partners who submit emergency department data to Maine CDC for syndromic surveillance. Currently, 24 of Maine's 37 emergency departments collect syndromic surveillance data and 20 of those receive a weekly syndromic surveillance report from Maine CDC. The survey was included with the report on August 14, 2012, and hospitals were given two weeks for completion. The survey included questions about how useful hospitals find syndromic surveillance and how data is shared back with the hospitals; which syndromes are most and least useful; and chief complaint data collection at individual hospitals.

\section{Results}

The survey was completed by 13 out of 22 emergency departments (59\% participation rate), and six out of 13 respondents ( $46 \%$ ) completed the entire survey. The factors reported as having an influence on a hospital's decision to submit data for syndromic surveillance were: public health importance of events (6 respondents) and assurance of privacy/confidentiality ( 5 respondents). The majority of respondents (5 respondents) reported that there are no factors that limit their ability to send emergency department data. Among hospitals that did report factors that limit their ability to send data, lack of information technology support in the hospital ( 2 respondents) and manually entering data/lack of electronic health records ( 1 respondent) were the most frequently reported. Six out of seven hospitals who answered (86\%) reported the current method of sharing syndromic surveillance data on a weekly basis, including a statewide data summary, as useful. Respondents also recommended that data be shared back with participants using 30-day line graphs for each syndrome (4 respondents). The three syndromes respondents found most useful were influenzalike illness ( 7 respondents), gastrointestinal (5 respondents), and respiratory ( 5 respondents). The three syndromes respondents found least useful were the broad heat syndrome (4 respondents), the narrow heat syndrome (4 respondents), and the other syndrome that captures all visits not classified into any syndrome (4 respondents). Chief complaint data, which is used to classify emergency department visits into syndromes, is most often recorded by a drop-menu (4 respondents)

\section{Conclusions}

With a low survey completion rate, it is difficult to generalize responses to all hospitals who participate in syndromic surveillance. Hospitals that did not respond to or complete the survey will be followed up with to determine their reasons for not doing so, as this may be useful information. In general, those who responded have more factors that influence them to contribute to syndromic surveillance than factors that hinder them. Most hospitals find the current method of sharing data back with the hospitals useful. Also, it is advantageous to know which syndromes the hospitals find most useful, as they are the entities that collect and report the data. Opinions differ among system users, which is why it is important to evaluate a system throughout all points of interaction.

Keywords

Syndromic surveillance; Evaluation; Acceptability

\section{Acknowledgments}

The authors would like to acknowledge those hospital partners who completed the survey.

\section{References}

Updated guidelines for evaluating public health surveillance systems. (2001) Morbidity and Mortality Weekly Report, 50(RR13);1-35. Retrieved

http://www.cdc.gov/mmwr/preview/mmwrhtml/rr5013a1.htm

\section{*Stefanie DeVita}

E-mail: stefanie.devita@maine.gov 